\title{
DIFICULTADES DE ESCRITURA EN EL CONTEXTO ESCOLAR CHILENO: ABORDAJE DE TERAPIA OCUPACIONAL Y LA UTILIZACION DE ESTRATEGIAS DE INTEGRACIÓN SENSORIAL
}

\author{
HANDWRITING DIFFICULTIES IN CHILEAN SCHOOL CONTEXT: OCCUPATIONAL \\ THERAPY INTERVENTION AND SENSORY INTEGRATION STRATEGIES
}

\section{Veronica Angulo D. ${ }^{1}$, Daniela Arenas M. ${ }^{2}$}

\section{Resumen}

La escritura y las dificultades de escritura son un tema importante para los Terapeutas Ocupacionales. La literatura evidencia que las dificultades de escritura constituyen uno de los motivos más frecuentes de derivación a Terapia Ocupacional (Feder et al., 2007)

En la investigación participan 25 Terapeutas Ocupacionales chilenos que trabajan en Programas de Inclusión Educativa (PIE) con niños de 6 a 9 años.

Los resultados indican que el $80 \%$ de la muestra considera que los problemas de escritura son un motivo frecuente de derivación a Terapia Ocupacional en el contexto escolar.

Las estrategias de Integración Sensorial para abordar los problemas de escritura en el contexto escolar son utilizadas en promedio por el $57 \%$ de los participantes, observándose en $3^{\circ}$ lugar en la frecuencia general, luego de la utilización de estrategias visomotoras y de estrategias volitivas/ motivacionales.

Las estrategias de Integración Sensorial son percibidas como las más efectivas para abordar los problemas de escritura en el contexto escolar en niños con Trastornos del Espectro Autista y Síndrome de Déficit Atencional, versus otras estrategias tales como las sensoriomotoras, volicionales / motivacionales, cognitivas o conductuales.

Las estrategias de integración sensorial más utilizadas para abordar los problemas de escritura en el contexto escolar, son las "sugerencias a profesores basadas en dicho enfoque", realizadas por el 87\% de los Terapeutas Ocupacionales participantes en el estudio.

\section{Palabras claves:}

Problemas de escritura, Integración Sensorial, Terapia Ocupacional en contexto escolar.

\begin{abstract}
Writing and handwriting difficulties are a major issue for Occupational Therapists. The literature shows that the handwriting difficulties are one of the most common reasons for derivation to Occupational Therapy (Feder et al., 2007)

Inthisresearch areparticipating 25chilean OccupationalTherapists whoseworkin InclusionEducationalPrograms (IEP) withchildren 6 to 9 years. Regarding the results; $80 \%$ believe that writing problems are a common reason derivation to Occupational Therapy in the school context. Sensory integration strategies to address handwriting problems in school context are used on average by 57\% of participants in the sample observed in 3rd place in the overall frequency after visual motor strategies of and volitional / motivational strategies. Sensory Integration strategies are perceived as the most effective in addressing the problems of writing in the school context in children with Autism Spectrum Disorder and Attention Deficit Disorder, versus other strategies such as sensorimotor, volitional / motivational, cognitive or behavioral. Sensory integration strategies most commonly used to address the problems of writing in the school context, are "suggestions to teachers" based on this approach, carried by $87 \%$ of occupational therapists in the study.
\end{abstract}

\section{Keywords:}

Handwriting problems, School Based Occupational Therapy, Sensory Integration.

1 Terapeuta Ocupacional Universidad de Chile, Magister Educación Superior Universidad Central de Chile. Certificación chilena de Especialidad en Integración Sensorial, Docente Escuela de Terapia Ocupacional Universidad Central de Chile vangulod@ucentral.cl

2 Estudiante de Terapia Ocupacional, Universidad Central, d.arenasmolina@gmail.com 


\section{INTRODUCCIÓN}

Una de las ocupaciones más importante en niños/as en edad pre-escolar y escolar es la Educación. Las escuelas proveen ambientes para una variedad de ocupaciones y los Terapeutas Ocupacionales apoyan a los niños y adolescentes para un desempeño satisfactorio en cada una de ellas. (Case-Smith, 2010)

En las últimas décadas y con el incremento de los Programas de Integración Escolar (PIE) en Chile, los Terapeutas Ocupacionales han asumido un desafío importante desarrollando la práctica y el posicionamiento de la Terapia Ocupacional en el contexto de educación regular. Este estudio pretende impulsar la reflexión respecto al razonamiento clínico y las decisiones de utilización de ciertos enfoques y estrategias para el abordaje de las necesidades ocupacionales que observamos frecuentemente en los niños/as en la escuela.

El abordaje de la Terapia Ocupacional en contexto escolar, frecuentemente incorpora varios aspectos (dependiendo de las necesidades del niño y sus familias), con el objetivo final de facilitar el desempeño en las diferentes actividades escolares y lograr la mayor participación posible al interior de la escuela.

Es posible visualizar al Terapeuta Ocupacional en diferentes acciones; apoyando por ejemplo en la adquisición de mayores grados de autonomía en actividades de autocuidado u otras de la rutina escolar, facilitando la participación en las diferentes actividades sociales y de recreo, adecuando materiales y utensilios escolares necesarios para el aprendizaje académico, prescribiendo y/o entrenando en tecnologías asistivas que faciliten la participación activa en clases, generando ambientes escolares inclusivos y facilitadores, trabajando en conjunto con educadoras y las familias, sensibilizando en la temática de discapacidad e inclusión social y/o apoyando al niño/a en el desarrollo de las habilidades motoras, sensoriales, cognitivas y sociales necesarias para el aprendizaje, dentro y fuera de la sala de clases.

Desde este contexto, una habilidad específica que permitirá la participación e involucramiento del niño/a en el contexto escolar y que requiere la adquisición de varias otras habilidades; la producción de escritura.

El Terapeuta Ocupacional frecuentemente observa el desempeño de los niños en sala de clases y diseña intervenciones para mejorar el desempeño escolar y las habilidades requeridas para el aprendizaje, las cuales pueden incluir el desarrollo de una escritura legible (Kiss, 2013)
La escritura la podemos definir como "la compleja habilidad perceptivo motora que abarca un conjunto de habilidades de coordinación motora, planeamiento motor, cognición y habilidades perceptivas, así como también sensibilidad táctil y kinestésica". (Feder et al, 2007). Las dificultades de escritura, sin una discapacidad neurológica o intelectual de base, es frecuentemente llamada disgrafía y típicamente incluye pobre legibilidad y baja velocidad de escritura (Feder et al., 2007), (Maeland, 1992).

Sabemos que el desarrollo de la comunicación escrita es una de las habilidades importantes requeridas en el contexto escolar. Alrededor del $30 \%$ al $60 \%$ de las actividades de un día de escuela, comprenden actividades de motricidad fina, tales como la escritura. La mayoría de estas actividades motoras finas (85\%) fueron tareas de papel y lápiz (McHale \& Cermak, 1992).

Pero, ¿Qué tan importante es la realización de una escritura legible?, aún cuando la incorporación del computador ha tomado importancia en las actividades escolares, la escritura aún sigue siendo una habilidad muy importante a desarrollar en los niños y por supuesto los profesores siguen enseñándola. La escritura es una habilidad esencial para permitir a los estudiantes expresar su conocimiento y pensamientos, así como también participar en numerosas actividades escolares. Aunque la mayoría de los estudiantes adquieren la habilidad de escritura, estudios refieren que aproximadamente del $10 \%$ al $20 \%$ de los niños en edad escolar presentan dificultades en este ámbito (Graham, 1996).

Un estudio dirigido a profesores evidencia que solo el $39 \%$ respondió que sus estudiantes escribían adecuadamente, el $46 \%$ no escribía lo suficientemente rápido como para cumplir con las demandas de la clase y que aproximadamente el $25 \%$ de los estudiantes experimentaban algún problema de escritura. (Smith, Holland \& Bishop, 2011).

Un estudio realizado por Karlsdottir \& Stefansson refiere que entre un $10 \%$ y $30 \%$ de los niños en edad escolar experimenta algún problema de escritura. (Karlsdottir \& Stefansson, 2002). Las dificultades en este ámbito podrían impactar directamente en bajo rendimiento escolar (Christensen, 2005), baja autoestima y en el sentimiento de efectividad en el sistema escolar (Kiss, 2013).

Respecto a la relación entre Terapia Ocupacional y los problemas de escritura, diversas publicaciones refieren que los problemas de escritura, son unos de los motivos más frecuentes de derivación a Terapia Ocupacional. 
(Feder et al., 2007). En Estados Unidos y Canadá, donde la mayoría de las escuelas públicas cuentan con un Terapeuta Ocupacional, éste es uno de los motivos más frecuentes de derivación y parte importante del quehacer del Terapeuta Ocupacional en el contexto escolar.

Los Terapeutas Ocupacionales ofrecen una variedad de estrategias para abordar los problemas de escritura en niños/as. Esta diversidad de estrategias tiene relación principalmente, con la diversidad de causas que podrían estar generando una dificultad de escritura. El razonamiento clínico llevará a los profesionales a priorizar la utilización del enfoque que más se adapta para dar solución a las dificultades específicas de cada niño/a. La modalidad de intervención podría ser individual en un contexto clínico, grupal en contexto escolar o podría dirigirse hacia el profesor/a o los padres.

En Canadá se realizó un estudio para conocer los diferentes enfoques teóricos que usaban los Terapeutas Ocupacionales para mejorar la escritura. Los hallazgos del estudio evidencian que existe una ecléctica orientación entre los Terapeutas Ocupacionales utilizando enfoques sensoriomotores $(90 \%)$, enfoques visomotores $(74 \%)$, enfoques de aprendizaje motor (68\%), enfoques cognitivos (64\%), enfoques biomecánicos (64\%), enfoques de integración sensorial (50\%) y enfoques de neurodesarrollo (42\%). El mismo estudio aplicado en Estados Unidos evidenció similares diversidad de enfoques (Feder et al, 2000)

La literatura describe que las intervenciones de Terapia Ocupacional desarrolladas para mejorar la escritura frecuentemente utilizan enfoques sensoriomotores que enfatizan el desarrollo de habilidades básicas para la escritura combinadas con un enfoque que enfatiza en la importancia de las habilidades cognitivas y habilidades ejecutivas (Weintraub et al, 2009)

La AOTA, refiere que las intervenciones de Terapia Ocupacional en niños/as con problemas de escritura podría incluir los siguientes aspectos: Demostrar la postura correcta y adecuada de los brazos, manos, cabeza y ojos, medir el nivel de fuerza y resistencia de miembro superior, analizar las habilidades motoras finas, determinar la habilidad de percepción visual que influye en la capacidad del niño para formar letras y formas, colaborar con los maestros en estrategias efectivas, proponer actividades para el hogar que promueven el desarrollo de habilidades necesarias para la escritura, entre otras. (AOTA, 2002)

\section{MÉTODO}

La presente investigación tiene un enfoque metodológico cuantitativo de diseño transversal. La técnica de muestreo a utilizar fue no probabilística por conveniencia, ya que se cuenta con una base de contactos de Terapeutas Ocupacionales que trabajan en contextos educativos en Chile. Los criterios de Inclusión fueron; Terapeutas Ocupacionales que trabajen en Proyectos de Integración Escolar (PIE), Terapeutas Ocupacionales que trabajen con niños en rango de edad de 6 a 9 años.

El instrumento de recolección de información utilizada, fue una encuesta que consta de 23 preguntas de modalidad abierta y cerrada. Las preguntas cerradas otorgan la posibilidad de agregar comentarios adicionales en caso que el profesional desee explayar su respuesta. La encuesta fue creada y subida a la plataforma Surveymonkey, herramienta gratuita para el desarrollo de encuestas online. Los datos se recogieron entre Octubre del 2015 y Diciembre 2015

El análisis de datos se realizó mediante un análisis de tipo estadístico descriptivo.

Respecto a las consideraciones éticas, las autoras se comprometen a no divulgar la identidad de los participantes y compartir con ellos los resultados del estudio. Todos asintieron explícitamente a participar. El estudio no genera riesgos asociados.

Respecto a la caracterización de la muestra, podemos referir lo siguiente:

- Participan 25 Terapeutas Ocupacionales, quienes cumplen con los requisitos de inclusión y asintieron al consentimiento informado.

- La mayoría de los Terapeutas Ocupacionales participantes trabajan en un establecimiento de tipo municipal-público (64\%).

- Respecto a los años de experiencia, la muestra tiene en promedio 3,2 años de experiencia. La mayoría de ellos tienen menos de 2 años de experiencia (52\%), el $88 \%$ tiene menos de 5 años de experiencia, siendo una muestra bastante joven, sin embargo representativa respecto a los que ocurre a nivel nacional.

- Respecto a la formación de pre y postgrado relacionado específicamente al tema de abordaje de Terapia Ocupacional en problemas de escritura, se observa que el $68 \%$ refiere no haber recibido formación en esta área durante el pregrado. Respecto al post-grado el $76 \%$ señala no haber recibido formación. 
Tabla 1:

Tipo de Establecimiento escolar en los que trabajan los T.O.

\begin{tabular}{|c|c|c|}
\hline Tipo de Establecimiento & No de T.O. $^{\text {Porcentaje }}$ \\
\hline Municipal-Publico & 16 & $64 \%$ \\
\hline Particular Subvencionado & 11 & $44 \%$ \\
\hline Privado & 1 & $4 \%$ \\
\hline
\end{tabular}

Tabla 2:

Años de experiencia de T.O. participantes en la muestra

\begin{tabular}{|c|c|c|}
\hline Años de Experiencia & No de T.O. & Porcentaje \\
\hline Entre 0 y 2 años & 13 & $32 \%$ \\
\hline entre 2 y 5 años & 9 & $12 \%$ \\
\hline más de 5 años & 3 & $100 \%$ \\
\hline TOTAL & 25 & 3,2 años \\
\hline Promedio & & \\
\hline
\end{tabular}

Tabla 3:

Formación de pregrado y postgrado en Abordaje de Terapia Ocupacional en Problemas de Escritura

\begin{tabular}{|c|c|c|c|c|c|}
\hline $\begin{array}{c}\text { Formación de } \\
\text { Pregrado }\end{array}$ & No de T.O. & $\%$ & $\begin{array}{c}\text { Formación de } \\
\text { Post-grado }\end{array}$ & No de T.O. & $\%$ \\
\hline SI & 8 & $32 \%$ & SI & 6 & 19 \\
\hline NO & 17 & $68 \%$ & NO & $25 \%$ & $76 \%$ \\
\hline Total & 25 & 100 & Total & 100 \\
\hline
\end{tabular}

\section{RESULTADOS}

El estudio presenta resultados en 6 aspectos:

1 Contexto de intervención y Percepción de problemas de escritura como un motivo frecuente de derivación en contexto escolar
Respecto a si los T.O. perciben los problemas de escritura como un motivo frecuente de derivación a Terapia Ocupacional en el contexto escolar, se observa lo siguiente: 
Tabla 4:

Percepción de motivo frecuente de derivación en contexto escolar

\begin{tabular}{|c|c|c|}
\hline Motivo frecuente de derivación & N $^{\circ}$ de T.O. & Porcentaje \\
\hline SI & 20 & $80 \%$ \\
\hline NO & 5 & $20 \%$ \\
\hline Total & 25 & $100 \%$ \\
\hline
\end{tabular}

El $80 \%$ cree que los problemas de escritura son un motivo frecuente de derivación a Terapia Ocupacional en el contexto escolar, mientras que un $20 \%$ cree que no es un problema de derivación frecuente. Esta percepción es similar a la encontrada por varios autores en evidencia internacional.

Tabla 5:

Contextos y modalidad de Intervención

\begin{tabular}{|c|c|c|}
\hline Contexto de Intervención & No de T.O. & Porcentaje \\
\hline Sala de clases & 0 & $0 \%$ \\
\hline Aula de recursos/ sala de T.O. & 12 & $48 \%$ \\
\hline Ambas & 13 & $52 \%$ \\
\hline Modalidad de Intervención & No de T.O. $^{\text {Porcentaje }}$ \\
\hline Individual & 9 & $36 \%$ \\
\hline Grupal & 0 & 0 \\
\hline Ambas & 16 & $64 \%$ \\
\hline
\end{tabular}

Respecto al contexto de intervención en el contexto escolar, se observa que el $48 \%$ realizaría la intervención en contexto de Aula de recursos o sala de Terapia Ocupacional (fuera de sala de clases) y que un $52 \%$ intervendría en ambos contextos; sala de clases y Aula de recursos / sala de Terapia Ocupacional.

Respecto a la modalidad de intervención, observamos que la mayoría $(64 \%)$ realizaría intervenciones de tipo individual y grupal para abordar los problemas de escritura. Un 36\% realizaría intervención solo de manera individual.

\section{2.- Diagnósticos frecuentes que reciben atención de Terapia Ocupacional en el contexto escolar}

Se pregunta a los T.O. que diagnósticos frecuentes tienen los niños con lo que trabajan en el contexto escolar. Se presentan los resultados respecto a los diagnósticos más frecuentes de los niños con los que trabajan los T.O. en el contexto escolar 
Tabla 6

Diagnósticos más frecuente en contexto escolar

\begin{tabular}{|c|c|c|}
\hline Diagnósticos & No de T.O. & Porcentaje \\
\hline TEL- Trastornos de Lenguaje & 9 & $36 \%$ \\
\hline TDAH / Síndrome de Déficit Atencional e Hiperactividad & 20 & $80 \%$ \\
\hline Trastorno de Aprendizaje (Digrafía u otros) & 11 & $44 \%$ \\
\hline Déficit Intelectual & 17 & $68 \%$ \\
\hline C.I. Rango limítrofe & 13 & $52 \%$ \\
\hline Trastornos de salud mental ( Trast. conductuales, trast. ansiosos, trast. animo) & 10 & $40 \%$ \\
\hline Trastornos del Espectro Autista & 22 & $88 \%$ \\
\hline Trastornos Motores & 16 & $64 \%$ \\
\hline Disfunciones de integración sensorial & 14 & $56 \%$ \\
\hline Retraso Desarrollo Psicomotor & 11 & $44 \%$ \\
\hline Déficit sensoriales (ceguera, baja visión, hipoacusia, etc.) & 7 & $28 \%$ \\
\hline
\end{tabular}

El 88\% refiere trabajar con niños con diagnóstico de Trastornos del Espectro Autista, siendo este el diagnóstico más frecuente en esta muestra. También aparece como diagnóstico frecuente el TDAH / Síndrome de Déficit Atencional e Hiperactividad (80\%) y el Déficit intelectual con un $68 \%$. Un porcentaje importante $(44 \%)$, refiere trabajar con niños con diagnósticos de Trastornos de Aprendizaje, donde formalmente se incluyen los problemas de escritura.

\section{3.- Percepción de diagnósticos frecuentes que presentan problemas de escritura en el contexto escolar}

Se presentan los resultados respecto a los diagnósticos que según la percepción de los Terapeutas Ocupacionales presentan frecuentemente problemas de escritura en el contexto escolar. Según su experiencia. ¿Cuáles de estos diagnósticos presentan frecuentemente problemas de escritura?

Tabla 7

Diagnósticos presentan frecuentemente problemas de escritura.

\begin{tabular}{|c|c|c|}
\hline Diagnósticos & $\mathrm{N}^{0}$ de T.O. & Porcentaje \\
\hline TEL- Trastornos de Lenguaje & 8 & $32 \%$ \\
\hline TDAH / Síndrome de Déficit Atencional e Hiperactividad & 14 & $56 \%$ \\
\hline Trastorno Especifico de Aprendizaje (Digrafía u otros) & 9 & $36 \%$ \\
\hline Déficit Intelectual & 13 & $52 \%$ \\
\hline C.I. Rango limítrofe & 2 & $8 \%$ \\
\hline Trastornos de salud mental ( Trast. conductuales, trast. ansiosos, trast. animo) & 1 & $4 \%$ \\
\hline Trastornos del Espectro Autista & 15 & $60 \%$ \\
\hline Trastornos Motores & 14 & $56 \%$ \\
\hline Disfunciones de integración sensorial & 7 & $28 \%$ \\
\hline Retraso Desarrollo Psicomotor & 8 & $32 \%$ \\
\hline Déficit sensoriales (ceguera, baja visión, hipoacusia, etc.) & 0 & $0 \%$ \\
\hline
\end{tabular}


Se observa que el $60 \%$ de los T.O. percibe que los niños con diagnósticos de Trastorno de Espectro Autista presentan problemas de escritura en el contexto escolar, siendo este diagnóstico el percibido con mayor frecuencia con problemas de escritura. También aparecen los diagnósticos de Trastornos motores (56\%), Síndrome de Déficit Atencional e Hiperactividad /TDAH (56\%) y el Déficit Intelectual (52\%)

\section{4.- Elección de estrategias para abordar problemas de escritura en el contexto escolar}

Para obtener la orientación general hacia un Enfoque o Marco de Referencia para la Intervención, se promediaron las estrategias de cada categoría. Los resultados se observan a continuación:

Tabla 8

Enfoques / Marcos / Modelos para abordar problemas de escritura

\begin{tabular}{|c|c|}
\hline $\begin{array}{c}\text { Enfoques/ Marcos / Modelos de Intervención para abordar problemas de escritura } \\
\text { (Promedio total de estrategias por enfoque) }\end{array}$ & Porcentaje \\
\hline Enfoque Visomotor & $70,4 \%$ \\
\hline Volicional- Modelo Ocupación Humana & $66,4 \%$ \\
\hline Enfoque Integración sensorial & $56,8 \%$ \\
\hline Modelo Biomecánico & $56 \%$ \\
\hline Modelos cognitivos & $55,2 \%$ \\
\hline Enfoque Control Motor & $53,6 \%$ \\
\hline Modelos conductuales & $50,6 \%$ \\
\hline Enfoques sensoriomotores & $50 \%$ \\
\hline Modelos compensatorias / rehabilitador & $48,8 \%$ \\
\hline
\end{tabular}

Los resultados nos pueden indicar que en general los T.O. utilizan una diversidad de estrategias para abordar problemas de escritura en el contexto escolar, no observándose diferencias significativas respecto a la elección de un enfoque sobre otro. Sin embargo se observa que el enfoque que en promedio muestra la mayor cantidad de utilización de estrategias es el enfoque visomotor $(70,4 \%)$.

A modo de referencia, en este estudio se describieron las siguientes estrategias visomotoras para el abordaje de problemas de escritura; pistas visuales para completar una letra ej: flechas de dirección, copiar modelos de letras desde una pizarra, copiar e imitar di- seños, Unión de puntos-Remarcar letras (sobre un modelo), colorear un área destinada sin sobrepasar límites, desarrollar habilidades de percepción visual (percepción de figura-fondo, discriminación visual, etc.)

Las estrategias de integración sensorial muestran un promedio de utilización de un 56,8\%, mostrándose en $3^{\circ}$ lugar en la preferencia general.

A continuación se observa un análisis de las diversas estrategias de manera independiente (no agrupadas por enfoque). Respecto a las diversas estrategias que utilizan los Terapeutas Ocupacionales para abordar los problemas de escritura en el contexto escolar: 
Tabla 9

Estrategias para abordar problemas de escritura

\begin{tabular}{|c|c|c|}
\hline Estrategias para abordar problemas de escritura & Nº de T.O. & Porcentaje \\
\hline Realizar la intervención en un contexto de juego / lúdico & 24 & $96 \%$ \\
\hline $\begin{array}{l}\text { Mantener una buena posición del cuerpo y del papel (favorecer el enderezamiento del tronco, } \\
\text { posicionamiento correcto de codos, línea media, disociación segmentaria, etc.) }\end{array}$ & 24 & $96 \%$ \\
\hline Engrosar lápices para facilitar el agarre & 23 & $92 \%$ \\
\hline Durante la intervención considerar intereses del niño y la motivación intrínseca & 22 & $88 \%$ \\
\hline Desarrollar habilidades de percepción visual (percepción de figura-fondo, discriminación visual, etc.) & 22 & $88 \%$ \\
\hline $\begin{array}{l}\text { Entregar sugerencias de integración sensorial a profesores que favorezcan las actividades de } \\
\text { escritura en niños }\end{array}$ & 21 & $84 \%$ \\
\hline Evitar sensación de fracaso, favorecer el sentido de logro & 21 & $84 \%$ \\
\hline Unión de puntos-Remarcar letras ( sobre un modelo) & 21 & $84 \%$ \\
\hline $\begin{array}{l}\text { Actividades sensoriales preparatorias: Antes de comenzar práctica de escritura, otorgar estimu- } \\
\qquad \text { lación táctil con masas, harina, semillas, etc. }\end{array}$ & 19 & $76 \%$ \\
\hline $\begin{array}{l}\text { Desarrollar habilidades de discriminación visual, detectando diferencia entre imagen de letras } \\
\text { y letras construidas. }\end{array}$ & 19 & $76 \%$ \\
\hline $\begin{array}{l}\text { Utilización de estimulación vestibular y propioceptiva conjunta para regular alerta, mejorar } \\
\text { postura, mejorar coordinación visomotora, etc. }\end{array}$ & 18 & $72 \%$ \\
\hline Permitir la utilización de letra imprenta v/s manuscrita & 18 & $72 \%$ \\
\hline $\begin{array}{l}\text { Desarrollar actividades que promuevan un patrón de agarre pulgar-índice-anular, fortaleci- } \\
\text { miento de la prensión pulgar-índice-anular, y actividades de una mano y estabilización }\end{array}$ & 18 & $72 \%$ \\
\hline
\end{tabular}

La estrategia "considerar contexto de juego / lúdico" $(96 \%)$, es la que se realizaría con mayor frecuencia, al igual que "mantener una buena posición del cuerpo y del papel (favorecer el enderezamiento del tronco, posicionamiento correcto de codos, línea media, disociación segmentaria, etc.) realizado con una frecuencia del $96 \%$.

La estrategia "engrosar lápices para facilitar el agarre", también aparece como una de las estrategias más frecuentes; el $92 \%$ de los T.O. la realizaría.

Respecto a la estrategia de Integración Sensorial más utilizada, se observa que "entregar sugerencias a profesores basadas en integración sensorial que favorezcan las actividades de escritura en niños" es utilizada por el $84 \%$ de la muestra.

\section{5.- Percepción de efectividad de estrategias para abordar problemas de escritura relacionado con diagnósticos en el contexto escolar}

Al observar la percepción de efectividad relacionado con los cuatro diagnósticos referidos como los atendidos con más frecuencia en el contexto escolar (Trastorno del Espectro Autista, Síndrome de Déficit Atencional, Déficit Intelectual, Trastornos motores), se observan las estrategias preferidas para abordar los problemas de escritura:

A continuación le pedimos nos indique que estrategias piensa Uds. son más efectivas para los siguientes diagnósticos: 
Tabla 10

Estrategias relacionadas con diagnósticos

\begin{tabular}{|c|c|c|c|c|}
\hline & $\begin{array}{c}\text { Estrategias } \\
\text { Sensoriomotoras }\end{array}$ & $\begin{array}{c}\text { Estrategias cog- } \\
\text { nitivas }\end{array}$ & $\begin{array}{c}\text { Estrategias volitivas/ } \\
\text { motivacionales }\end{array}$ & $\begin{array}{c}\text { Estrategias de Inte- } \\
\text { gración Sensorial }\end{array}$ \\
\hline $\begin{array}{c}\text { TDAH-Síndrome de Déficit Atencional } \\
\text { e Hiperactividad }\end{array}$ & $24 \%$ & $4 \%$ & $12 \%$ \\
\hline Déficit Intelectual & $16 \%$ & $36 \%$ & $8 \%$ & $12 \%$ \\
\hline Trastornos del Espectro Autista & $8 \%$ & $4 \%$ & $8 \%$ & $08 \%$ \\
\hline Trastornos Motores & $32 \%$ & $0 \%$ & $0 \%$ & $7 \%$ \\
\hline PROMEDIO & $20 \%$ & $11 \%$ & & $28 \%$ \\
\hline
\end{tabular}

Al observar el promedio general, las estrategias de Integración Sensorial son las percibidas como las más efectivas para abordar los problemas de escritura ( $28 \%)$, seguidas por las sensoriomotoras $(20 \%)$ y las estrategias cognitivas $(11 \%)$ en los diagnósticos atendidos con mayor frecuencia por la muestra.

Al analizar las estrategias utilizadas aisladas por diagnóstico, se observa lo siguiente:

Trastorno de Espectro Autista: Es el diagnóstico que presenta mayores diferencias y menos dispersión de estrategias. Las estrategias de Integración sensorial son percibidas por el $68 \%$ de la muestra como las más efectivas para abordar los problemas de escritura respecto a otros tipos de estrategias tales como las conductuales, sensoriomotoras, cognitivas y motivacionales.

Trastorno de Déficit Atencional e Hiperactividad: Lo mismo ocurre con el diagnóstico de TDAH, donde las estrategias de Integración sensorial también muestran mayor frecuencia de respuestas. Estrategias de Integración sensorial son percibidas por el $32 \%$ de la muestra como las más efectivas para abordar los problemas de escritura respecto a otros tipos de estrategias tales como las conductuales, sensoriomotoras, cognitivas y motivacionales.

\section{6.- Tipo de estrategias de Integración Sensorial utilizadas para el abordaje de problemas de escritura en el contexto escolar.}

Respecto a cuales son las estrategias de Integración sensorial en general utilizadas con más frecuencia para abordar los problemas de escritura en el contexto escolar, se observa:

Tabla 11

Estrategias de Integración Sensorial en el contexto escolar

\begin{tabular}{|c|c|c|}
\hline Estrategias de Integración Sensorial & No de T.O. & Porcentaje \\
\hline $\begin{array}{c}\text { Utilización de lápices de diversas texturas y tamaños ( por ej. lápices vibradores, } \\
\text { lápices con peso, etc.). }\end{array}$ & 17 & $68 \%$ \\
\hline $\begin{array}{c}\text { Utilización de estimulación vestibular y propioceptiva conjunta para regular alerta, } \\
\text { mejorar postura, mejorar coordinación visomotora, etc. }\end{array}$ & 18 & 10 \\
\hline $\begin{array}{c}\text { Utilización de cojines de semilla o de agua, cambiar silla tradicional por pelota, maní } \\
\text { terapéutico u otro para favorecer integración sensorial en actividades de escritura. }\end{array}$ & $52 \%$ \\
\hline Incluir peso, mochila de peso durante las actividades de escritura & 5 & $20 \%$ \\
\hline $\begin{array}{c}\text { Entregar sugerencias a profesores que favorezcan las actividades de escritura en niños } \\
\text { con problemas de integración sensorial. }\end{array}$ & $84 \%$ \\
\hline
\end{tabular}


Respecto a las estrategias de integración sensorial utilizadas en el contexto escolar, observamos en primera preferencia $(87,50 \%)$ las "sugerencias a profesores basadas en integración sensorial" para mejorar escritura en niños/as", la "utilización de estimulación vestibular y propioceptiva conjunta para regular alerta, mejorar postura, mejorar coordinación visomotora, etc." (75\%) y la "utilización de lápices de diversas texturas y tamaños ( por ej. Lápices vibradores, lápices con peso, etc.)", utilizados por el 70,83\%. La estrategia menos utilizada fue "incluir peso, mochila de peso durante las actividades de escritura" utilizado solo por el 20,83\% .

\section{Discusión y CONCLUSIONES}

Se presentan las siguientes conclusiones:

- La literatura demuestra que los Terapeutas Ocupacionales apoyan el desarrollo de las habilidades para la escritura y generan estrategias que podrían resultar en un abordaje eficaz para las dificultades de escritura en niños y niñas.

- Respecto a la formación profesional tenemos un gran desafío; el estudio muestra que existe una escasa formación tanto de pre-grado como de postgrado en el abordaje específico de Terapia Ocupacional en niños con problemas de escritura y que sin embargo este constituye un motivo frecuente de derivación en el contexto escolar ( $80 \%$ indica que este constituye problema frecuente de derivación)

- La mayoría de los Terapeutas Ocupacionales (52\%) abordan los problemas de escritura en contextos de sala de clases y Aula de recursos / sala de Terapia Ocupacional de manera complementaria.

- Respecto a la modalidad de intervención, observamos que la mayoría (64\%) realizaría intervenciones de tipo individual y grupal (ambas) para abordar los problemas de escritura. Un 36\% realizaría intervención solo de manera individual

- La diversidad de enfoques utilizados para abordar los problemas de escritura observados en este estudio, se condice con lo que demuestra la evidencia a nivel internacional, donde se refiere que los T.O. utilizarían diversidad de enfoques. (Feder 2000) o que incluso varios enfoques pudieran utilizarse en una misma sesión.

- Respecto a cuales serían los enfoques más utilizados para abordar problemas de escritura, según el estudio de Feder en Canadá y EEUU (Feder 2000) sería el enfoque sensoriomotor $(90 \%)$ el utilizado con más frecuencia. Integración sensorial mostraría una utilización del 50\%. En el presente estudio observamos que las estrategias visomotoras son las que utilizarían los T.O. con mayor frecuencia $(70 \%)$, las estrategias de tipo volicionales/ motivacionales $(66 \%)$ y las de integración sensorial $(57 \%)$ se muestran en $3^{\circ}$ orden.

- Respecto a la elección de estrategias asociadas por diagnóstico se observa que las estrategias de Integración sensorial son percibidas por el $68 \%$ de la muestra como las más efectivas para abordar los problemas de escritura en niños con diagnóstico de Trastorno de Espectro Autista, en comparación a otros tipos de estrategias tales como las conductuales, sensoriomotoras, cognitivas y motivacionales. Otro aspecto interesante resulta en que este diagnóstico presenta la menor dispersión de estrategias.

- Lo mismo ocurre con el diagnóstico de Trastorno de Déficit Atencional e Hiperactividad / TDAH, donde las estrategias de Integración sensorial también muestran mayor frecuencia de respuestas. Estrategias de Integración sensorial son percibidas por el $32 \%$ de la muestra como las más efectivas para abordar los problemas de escritura respecto a otros tipos de estrategias tales como las conductuales, sensoriomotoras, cognitivas y motivacionales.

- Respecto a las 10 estrategias más utilizadas en general para abordar problemas de escritura en contexto escolar aparecen estrategias motivacionales tales como "Realizar la intervención en un contexto de juego / lúdico" ( 96\%), considerar intereses del niño y la motivación intrínseca (88\%), evitar sensación de fracaso, favorecer el sentido de logro (84\%) y algunas otras estrategias como "mantener una buena posición del cuerpo y del papel (favorecer el enderezamiento del tronco, posicionamiento correcto 
de codos, línea media, disociación segmentaria, etc.) $(96 \%)$, "engrosar lápices para facilitar el agarre" $(92 \%)$ y desarrollar habilidades de percepción visual (percepción de figura-fondo, discriminación visual, etc.) $(88 \%)$

- La estrategia de Integración sensorial que figura dentro de las 10 más frecuentes, es "sugerencias a profesores basadas en integración sensorial para favorecer las actividades de escritura en niños" desarrollada por el $84 \%$ de los T.O. para abordar problemas de escritura en contexto escolar.

- Adicionalmente es importante mencionar el antecedente que nos proporciona el catastro 2015 de la REDTOEDUCACION la cual nos indica que de una muestra de 165 Terapeutas Ocupacionales chilenos que trabajan en contextos educativos, tales como Jardines infantiles, Escuelas regulares, Programas de Inclusión Educativa, Escuelas Especiales, entre otros, un número importante de ellos ( $47,2 \%$ ) refiere tener alguna formación de postítulo en Integración Sensorial, siendo este sin duda la formación de postgrado más frecuente con la que cuentan los Terapeutas Ocupacionales chilenos que se desempeñan en el contexto escolar. El fenómeno anterior, podría generar que más Terapeutas Ocupacionales en Chile utilicen Integración sensorial como un enfoque para el abordaje de problemas de escritura en el contexto escolar.

- Finalmente el estudio nos entrega antecedentes respecto a que Integración Sensorial constituye un enfoque de gran influencia en la práctica de la Terapia Ocupacional en contextos educativos y uno de los más utilizados por los Terapeutas Ocupacionales para abordar los problemas de escritura en el contexto escolar chileno.

\section{AgRADECIMIENTOS}

Agradecemos a los Terapeutas Ocupacionales que participaron en el estudio, Maria Ignacia Meza, Macarena Carrasco, Daniela Zuñiga (Estudiantes de Terapia Ocupacional Universidad Central) y a la REDTOEDUCACION Chile.

\section{RefERENCIAS BibliogRÁfiCAS}

Asociación Americana de Terapia Ocupacional (2002). Marco de trabajo para la práctica de la terapia ocupacional: ámbito de dominio y proceso. ( $2^{\mathrm{a}} \mathrm{a}$ ed.).

Batya, E. (2012) Age effects on sensory-processing abilities and their impact on handwritin.g Revue canadienne d'ergothérapie.

Case-Smith, J., Holland, T., \& Bishop, B. (2011). Effectiveness of an integrated handwriting program for first-grade students: A pilot study. American Journal of Occupational Therapy, 65, 670-678. doi: 10.5014/ajot.2011.000984.

Case-Smith y O'brien. (2010). Occupational Therapy for children, $6^{\circ}$ edition. EEUU: Mosby.

Christensen, C. (2005). The Role of ortographic motor integration in the prodution of creative and well estrucutres writen text for student in secondary school. Educational Psicologhy, 441-453.

Colegio de Terapeutas Ocupacionales de Navarra (COTONA). (2006). Terapia Ocupacional en educación. Recuperado de: http://www. terapia-ocupacional.com/articulos/Educacion_Terapia_ocupacional_Navarra_06.pdf.

De la Cruz, M., Scheuer, N., Baudino, V., Huarte, M., Sola, G., \& Pozo, J. (2002). ¿Cómo aprenden a escribir los niños? Las concepciones de padres y maestros de comunidades educativas en entornos socioculturales medios marginados. Estudios pedagógicos (Valdivia), (28), 7-29. Recuperado en 09 de junio de 2015, de http://www.scielo.cl/scielo.php?script=sci_arttext\&pid=S071807052002000100001\&lng=es\&tlng=es. 10.4067/S071807052002000100001.

Díaz, C., \& Price, M. (2012). ¿Cómo los niños perciben el proceso de la escritura en la etapa inicial?. Estudios pedagógicos (Valdivia), 38(1), 215-233. Recuperado en 09 de junio de 2015, de http://www.scielo.cl/scielo.php?script=sci_arttext\&pid=S0718$\underline{07052012000100013 \& \operatorname{lng}=\mathrm{es} \& t \operatorname{lng}=\mathrm{es} . \quad 10.4067 / \mathrm{S} 0718-}$ 07052012000100013.

Feder, K., Majnermer, A., \& Synnes, A. (2000). Handwriting: Current trends in occupational therapy practice. Canadian Journal of Occupational Therapy , 197-204.

Feder, K. \& Majnemer, A. (2007). Handwriting development, competency, and intervention. Development Medicine and Child Neurology, 312-317.

Feder, k., Majnemer, A., Bourbonnais, D., Blayney, M., \& Morin, I. (2007). Handwriting performance on the ETCH-M of students in a grade one regular education program. Occupational Therapy in Pediatrics, 27, 43-62.

Graham, S. \& Weintraub, S. (1996). A review of Handwriting reserch : Progress and prospects. Educational Psicologhy Review, 8-87.

Hoy, M., Egan, M., \& Feder, K. (2011). A systematic review of interventions to improve handwriting. Canadian Journal of Occupational Therapy,78, 13-25. doi: 10.2182/cjot.2011.78.1.3 
Jill G. Zwicker, Allyson F. Hadwin (2007) Cognitive Versus Multisensory Approaches to Handwriting Intervention: A Randomized Controlled Trial. OTJR: Occupation, Participation and Health.

Karlsdottir, R., \& Stefansson, T. (2002). Problems in developing functional handwriting . Percept Motor Skills, 623-662.

Kiss, D. (2007). "Handwriting consultation in elementary schools" OT Practice, 12 (14), 11-14.

Kiss, D. (2013). Handwriting Consultation in Elementary Schools. OT Practice.

Lust, C., \& Donica, D. (2011). Research Scholars Initiative-Effectiveness of a handwriting readiness program in Head Start: A two-group controlled trial. American Journal of Occupational Therapy, 65, 560-568. doi: 10.5014/ ajot.2011.000612

Maeland, A. (1992). Handwriting and perceptual-motor skills in clumsy, dysgraphic, and "normal" children. Perceptual and Motor Skills, 75, 1207-1217.

Marr, D. (2005). Consistency of Handwriting Performance Across the Early Elementary Grades. Occupation, Participation and Health, 25 (4), 143-148. doi: 10.1177/153944920502500403OTJR

McHale, K., \& Cermak, S. (1992). Fine motor activities in elementary school: Preliminary findings and provisional implications for children with fine motor problems. American Journal of Occupatinal Therapy, 46, 898-903.

Ministerio de Educación. (2010). Orientaciones para la implementación del decreto $N^{\circ} 170$ en programas de integración escolar. Santiago: División de Educación General. Recuperado de http://www. mineduc.cl/usuarios/edu.especial/file/documentos\%202010/formularios\%20unicos/orientaciones_implementacion_dto170_pie_ v2.pdf

Ministerio de Educación. (2009). Decreto con toma de razón nº170. Santiago. Recuperado de: http://www.mineduc.cl/usuarios/edu. especial/doc/201502131253220.Decreto170.pdf

Ministerio de Educación. (2009). Programa de Integración Escolar PIE. Manual de orientaciones y apoyo a la gestión. (Directores y sostenedores). Recuperado de: http://www.mineduc.cl/usuarios/edu. especial/doc/201405071255480.ManualOrientacionesPIE.pdf

Ministerio de Educación. (2013). Orientaciones Técnicas para Programas de Integración Escolar (PIE). Recuperado de: http://www.mineduc. cl/usuarios/edu.especial/doc/201310081640100.orientacionesPIE2013.pdf

Rigal, R. (2006). Educación Motriz y educación psicomotriz en Preescolar y Primaria. Barcelona: Inde.

Rocha E., Luiz, A., \& Zulian M. Refexões sobre as possíveis contribuições da terapia ocupacional nos processos de inclusão escolar. Revista de Terapia Ocupacional da Universidade de São Paulo. 2003; 14(2): 72-78.

VV.AA. (1998): Fundamentos de Educación Física para enseñanza Primaria, 2 volúmenes. Barcelona: INDE.
Weintraub, N., Yinon, M., Hirsch, I., \& Parush, S. (2009). Effectiveness of sensoriomotor and task-oriented handwriting intervention in elementary school-aged students with handwriting difficulties. OTJR: Occupation, participation and health, 125-134.

Weintraub, N. (2008). Effectiveness of Sensorimotor and Task-Oriented Handwriting Intervention in Elementary School-Aged Students with Handwriting Difficulties. OTJR: Occupation, Participation and Health.

Zachry, A. H. (2011). Sensory writing: A team approach to handwriting instruction. OT Practice, 16, 7-8. 